\title{
Enthalpy Landscape Dictates the Irradiation-Induced Disordering of Quartz ${ }^{\dagger}$
}

\author{
N. M. Anoop Krishnan, ${ }^{1}$ Bu Wang, ${ }^{1,2}$ Yingtian Yu, ${ }^{1}$ Yann Le Pape, ${ }^{3}$ Gaurav Sant, ${ }^{2,4}$ and Mathieu Bauchy ${ }^{1, *}$ \\ ${ }^{1}$ Laboratory for the Physics of Amorphous and Inorganic Solids (PARISlab), Department of Civil and \\ Environmental Engineering, University of California, Los Angeles, California 90095, USA \\ ${ }^{2}$ Laboratory for the Chemistry of Construction Materials $\left(L C^{2}\right)$, Department of Civil and Environmental \\ Engineering, University of California, Los Angeles, California 90095, USA \\ ${ }^{3}$ Oak Ridge National Laboratory, P.O. Box 2008, Oak Ridge, Tennessee 37831-6148, USA \\ ${ }^{4}$ California Nanosystems Institute (CNSI), University of California, Los Angeles, California 90095, USA
}

(Received 9 January 2017; revised manuscript received 14 April 2017; published 28 July 2017)

Under irradiation, minerals tend to experience an accumulation of structural defects, ultimately leading to a disordered atomic network. Despite the critical importance of understanding and predicting irradiationinduced damage, the physical origin of the initiation and saturation of defects remains poorly understood. Here, based on molecular dynamics simulations of $\alpha$-quartz, we show that the topography of the enthalpy landscape governs irradiation-induced disordering. Specifically, we show that such disordering differs from that observed upon vitrification in that, prior to saturation, irradiated quartz accesses forbidden regions of the enthalpy landscape, i.e., those that are inaccessible by simply heating and cooling. Furthermore, we demonstrate that damage saturates when the system accesses a local region of the enthalpy landscape corresponding to the configuration of an allowable liquid. At this stage, a sudden decrease in the heights of the energy barriers enhances relaxation, thereby preventing any further accumulation of defects and resulting in a defect-saturated disordered state.

DOI: 10.1103/PhysRevX.7.031019

Subject Areas: Chemical Physics,

Computational Physics,

Materials Science

\section{INTRODUCTION}

Understanding irradiation-induced damage is of great importance for applications including nuclear waste immobilization, nuclear plant safety, and nuclear fuel form integrity, as well as for the controlled doping of semiconductors and in fusion reactors [1-5]. Irradiation induces the formation and accumulation of defects in crystals' atomic networks $[1,6]$. Eventually, this can result in a loss of long-range order in the network, although some degree of short-range order is preserved, e.g., interatomic distances or coordination numbers [6-9]. This is similar to what is

\footnotetext{
The United States Government retains and the publisher, by accepting the article for publication, acknowledges that the United States Government retains a nonexclusive, paid-up, irrevocable, worldwide license to publish or reproduce the published form of this manuscript, or allow others to do so, for United States Government purposes. The Department of Energy will provide public access to these results of federally sponsored research in accordance with the DOE Public Access Plan (http://energy.gov/downloads/doe-public-access-plan).

*Corresponding author. bauchy@ucla.edu

Published by the American Physical Society under the terms of the Creative Commons Attribution 4.0 International license. Further distribution of this work must maintain attribution to the author(s) and the published article's title, journal citation, and DOI.
}

observed in conventional glasses, i.e., when materials are quenched from the liquid state fast enough to avoid crystallization [10]. After exposure to a critical radiation energy, the amount of defects saturates, and no further changes in the structure are observed [6]. Although a glass could be assumed to represent the upper (structural) limit of irradiation-induced disordering, glasses themselves can also show damage upon irradiation [9,11]. In addition, such comparisons are not obvious as glassy materials are out of equilibrium; as such, their properties depend on their thermal history [12]. As a result, several questions remain unclear $[1,2]$, including the following: (1) Does irradiation-induced disordering fundamentally differ from the amorphization that is induced via heating and fast quenching? (2) Does defect saturation occur when the system reaches a glassy state?

Numerous empirical and phenomenological models have been proposed to answer these questions [13]. For example, the resistance to irradiation of materials has been shown to be related, amongst others, to the atomic structure $[3,14]$ and topology [15-17], the ability of the network to accommodate lattice disorder [2,18], bond energies [19], glass-forming ability [20], melting point [21], or other physical properties [22]. In turn, the saturation of damage (defects) observed at high dosage has been suggested to arise when the network loses its crystalline order $[6,13]$ or becomes unable to accommodate defect-induced swelling [23]. Nevertheless, it is widely acknowledged that none of these models can simultaneously explain all observations $[1,5]$. 
Taking an alternative viewpoint, all of these properties ultimately depend on the enthalpy landscape of the material. Hence, such a framework can provide a unifying and physically sound basis to describe irradiation effects [1]. Here, via reactive molecular dynamics (RMD) simulations of $\alpha$-quartz as an example mineral, we demonstrate the crucial role of the topography of the enthalpy landscape in controlling irradiation-induced amorphization. By characterizing the different configurations of the enthalpy landscape that are accessed upon irradiation, we show that, prior to defect saturation, irradiated quartz explores "forbidden states" of the enthalpy landscape, i.e., those that cannot be achieved by simply cooling a silica melt. Importantly, we establish that irradiation-induced disordering saturates when the system achieves the atomic configuration of an "allowable liquid," whose enthalpy landscape features low-energy barriers, thereby facilitating the relaxation of defects and preventing any further damage.

\section{METHODS}

\section{A. Irradiation simulations}

Realistic RMD simulations of irradiation-induced damage in $\alpha$-quartz are carried out using LAMMPS [24]. To this end, we follow a well-established methodology $[6,16,25,26]$. First, a randomly chosen atom is accelerated with a kinetic energy equivalent to that of the targeted incident neutron. Here, an incident energy of $600 \mathrm{eV}$ is used. Note that weighted probabilities based on the neutron cross sections of silicon and oxygen atoms are considered when choosing the incident atom. Once the atom is accelerated with the desired incident energy, it collides with other atoms, thereby resulting in a ballistic cascade. A spherical region is then created around the impacted zone, outside which atoms are kept at a constant temperature of $300 \mathrm{~K}$ by a Nosé-Hoover thermostat [27]. In contrast, to avoid any spurious effects of the thermostat on the dynamics of the cascade, the dynamics of the atoms inside the sphere is treated in the $N V E$ ensemble. The radius of the $N V E$ sphere is fixed as $10 \AA$. Note that a variable time step is used during the ballistic cascade to avoid potential numerical errors associated with excessive collisions and overlapping of atoms due to the high velocities of the primary and secondary knock-on atoms; a time step of $0.5 \mathrm{fs}$ is used otherwise. The dynamics of the cascade is simulated for $20 \mathrm{ps}$, which was found to be long enough to ensure the convergence of both temperature and energy. Finally, after each collision, the system is further relaxed in the $N P T$ ensemble at $300 \mathrm{~K}$ and zero pressure for another 5 ps. This enables the system to adjust its density upon irradiation. Such an iterative process is then repeated, with different knock-on atoms, until the system exhibits a saturation in terms of both enthalpy and density.

The irradiation-induced amorphization can occur via different mechanisms [28,29], e.g., accumulation of point defects [30], interface-controlled amorphization [31], multiple cascade overlap [32], in-cascade amorphization [33], or direct impact [29]. In most cases, amorphization is likely to occur through a combination of these processes. However, the final irradiated state does not significantly depend on the specific mechanism of amorphization $[28,29,34,35]$. However, it should be noted that there are certain caveats associated with MD simulations of radiation damage. On the one hand, because of the limited time scale accessible to MD simulations, the damage flux imposed here is higher than those typically observed in radiation experiments. On the other hand, the incident energies used in our radiation simulations $-300 \mathrm{eV}, 600 \mathrm{eV}$, and $1000 \mathrm{eV}$ (see Ref. [36]) - are lower than experimental values, which can be of the order of 10-100 keV. However, although the simulations represent time scales lower than experiments, because of the infinitesimally low diffusion coefficient of oxygen atoms in quartz and silica at low temperatures [28], structural defects are unlikely to migrate through the system, even over extended periods of time.

Because of the large region that is affected during each ballistic cascade, large system sizes are required to avoid potential spurious self-interactions arising from the periodic boundary conditions. Herein, the system size is determined by repeatedly knocking each atom of the quartz primitive cell, with the target radiation energy and in random directions. The maximum distances traveled by each of the impacted atoms are then recorded. We eventually choose the size of the system to be at least twice as large as the maximum distance among all the recorded ones. In the present case, the initial system consists of a $10 \times 10 \times 9 \alpha$-quartz supercell comprising 8100 atoms. It is worth noting that, to offer realistic results, RMD simulations require the use of accurate interatomic potentials. In particular, in the case of irradiation simulations, the interatomic potential must (1) correctly describe both the pristine and disordered structures of the relevant system with a fixed set of parameters, (2) provide a realistic description of high-energy collisions, wherein pairs of atoms potentially explore the short-distance part of the potential, and (3) be able to handle the formation of atomic species with defective local environments-e.g., overcoordinated or undercoordinated atoms - which are likely to form upon irradiation. To this end, we use the ReaxFF potential [29], with parameters taken from Manzano et al. [30], as it can correctly describe the structure of both pristine $\alpha$-quartz and glassy silica and features robust potential forms that can dynamically adjust the potential energy based on the local atomic environment of atoms [31]. Furthermore, thanks to the shielding of shortrange interactions [32,33], ReaxFF can be used without any modifications to simulate high-energy collisions and ion bombardment.

\section{B. Glass preparation}

In order to compare the structure of irradiated quartz to that of its glassy counterpart, a silica glass of similar size 
(8100 atoms) is prepared using RMD simulations following the melting-quenching method [31]. Note that, to ensure a meaningful comparison with irradiated quartz, we rely on the same potential and timestep. First, an initial system is generated by randomly placing atoms in a cubic box, while ensuring the absence of any unrealistic overlap. The system is then melted at $4500 \mathrm{~K}$ at zero pressure for $1 \mathrm{~ns}$ in the $N P T$ ensemble, which ensures that the memory of the initial configuration is lost. The system is subsequently gradually cooled from $4500 \mathrm{~K}$ to $300 \mathrm{~K}$ at zero pressure with a cooling rate of $1 \mathrm{~K} / \mathrm{ps}$ in the $N P T$ ensemble. The formed glass is finally equilibrated at $300 \mathrm{~K}$ and zero pressure for $1 \mathrm{~ns}$ in the NPT ensemble to ensure complete relaxation of the structure. Note that, although the cooling rate can significantly affect the thermodynamic conditions of glassy silica such as its density, it weakly affects its short- and medium-range order structure $[37,38]$. In particular, the density of glassy silica can vary from $2.3 \mathrm{~g} / \mathrm{cm}^{3}$ to $2.2 \mathrm{~g} / \mathrm{cm}^{3}$ depending on the cooling rate [37,38]—-lower cooling rates yielding lower densities. However, the computational cost of reactive potentials like ReaxFF prevents the usage of cooling rates that are significantly lower than the one used herein. The resulting density of silica glass is found to be slightly overestimated, around $2.3 \mathrm{~g} / \mathrm{cm}^{3}$. However, the final structure of the simulated glass shows a good agreement with experimental data [31].

\section{Ground-state enthalpy computation}

In this study, we aim to meaningfully compare the enthalpy of irradiated quartz to that of glassy silica. However, if calculated at finite temperature using RMD, the energy of the system comprises the contributions of the random vibrations of the atoms. As such, the random sampling of the configurational space will result in some uncertainty in the instantaneous potential energy of the system. To overcome this issue, starting from the configurations obtained at a given temperature, we compute the ground-state enthalpy, that is, the enthalpy of the inherent structure. This is achieved by performing an energy minimization while enforcing a zero pressure, following the method presented elsewhere [34]. This ensures that all atoms reach a local minimum of potential energy, thereby removing any thermal contribution from the computed enthalpy. Note that this method provides the local-i.e., not absolute-minimum of enthalpy of the system and, as such, can be used to obtain the value of the ground-state enthalpy at a given temperature. Such evolutions are investigated, both for $\alpha$-quartz and glassy silica, by the following procedure. Starting from structures equilibrated at $300 \mathrm{~K}$ and zero pressure, the system is gradually heated at a rate of $1 \mathrm{~K} /$ ps under zero pressure in the $N P T$ ensemble up to a temperature of $4500 \mathrm{~K}$, that is, when both the crystal and glass melt. A posteriori, independent atomic configurations are selected every $100 \mathrm{~K}$, instantaneously cooled to $1 \mathrm{~K}$, and further relaxed for $50 \mathrm{ps}$ at this temperature in the $N P T$ ensemble. The ground-state enthalpy is eventually computed for each configuration.

\section{Ring-size distribution}

The distribution of Si-O ring sizes in irradiated quartz, liquid silica, and glassy silica is computed using the RINGS package [35], wherein, starting from a given $\mathrm{Si}$ atom, a ring is defined as the shortest closed path that comes back to this atom. The size of the ring is then defined by the number of connected Si atoms. Therefore, a ring size of $n$ contains a total of $2 n$ atoms, where $n$ corresponds to the number of $\mathrm{Si}$ (or $\mathrm{O}$ ) atoms. The cutoff used to define an active $\mathrm{Si}-\mathrm{O}$ bond $(2.1 \AA)$ is obtained from the partial PDF. A maximum ring search size of 20 is used (the convergence of the ringsize distribution was ensured by considering higher maximum ring sizes).

\section{E. Roughness of the local enthalpy landscape}

For a given configuration, the height of the energy barriers at the vicinity of the local equilibrium position of the system is estimated using the following methodology. First, we perform multiple energy minimizations on the pristine, partially irradiated, and fully irradiated quartz samples. This is to ensure that the samples are at their ground state, corresponding to a temperature of $0 \mathrm{~K}$. Then, we provide a sudden energy bump to the system corresponding to a temperature of $1500 \mathrm{~K}$. The temperature is chosen to be high enough to allow potential motion between low-energy barriers but low enough to avoid glass transition or melting of the system. Finally, we allow the system to evolve in the microcanonical ensemble $(N V E)$ for 100 ps. The MSD $\left(r^{2}\right)$ is obtained from $r^{2}=(1 / N) \sum_{i=1}^{N}\left(\overrightarrow{r_{i}}-\overrightarrow{r_{i, 0}}\right)^{2}$, where $N$ is the number of atoms, $r_{i}$ are the positions of each atom after $100 \mathrm{ps}$ of dynamics following the energy bump, and $r_{i, 0}$ are the positions in the inherent structure $(0 \mathrm{~K})$.

\section{RESULTS}

To establish our conclusions, we rely on RMD simulations, which allow us to assess the effect of irradiation on the structure and enthalpy landscape of $\alpha$-quartz. The specifics of the simulations are provided in Sec. II. Figure 1(a) shows the evolution of the enthalpy and density of $\alpha$-quartz upon irradiation. First, we observe that the density of quartz decreases monotonically upon irradiation and eventually converges to around $2.2 \mathrm{~g} / \mathrm{cm}^{3}$, in agreement with experimental data $[7,8,39,40]$. Second, we note that the enthalpy increases upon irradiation, before plateauing. This arises from the formation and accumulation of energetically unfavorable structural defects in the atomic network, as presented elsewhere [25]. Note that, here, any deviation from the initial pristine structure is referred to as a defect, which somewhat differs from the typical crystallographic viewpoint of "defects," e.g., point, interstitial, 
(a)

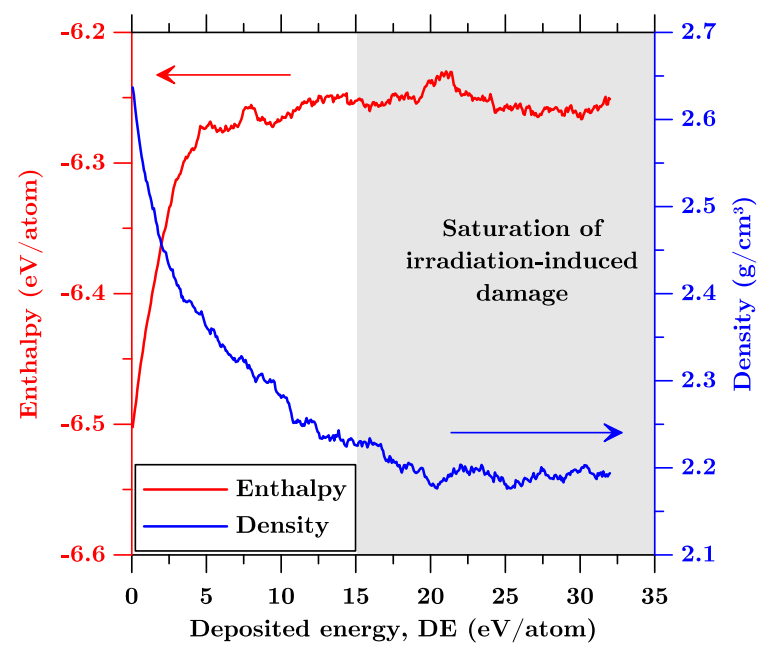

(b)

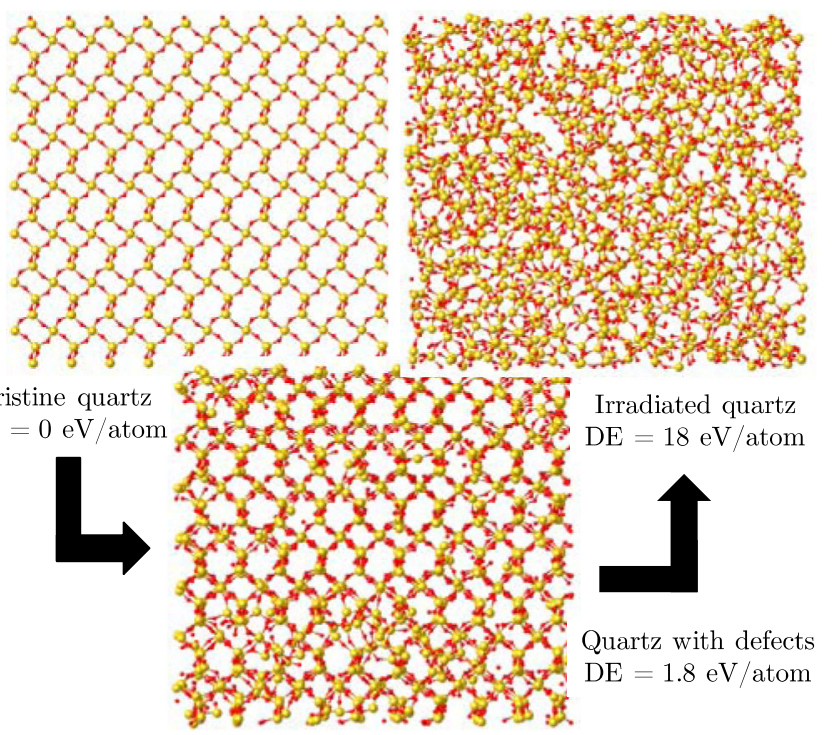

FIG. 1. Irradiation-induced disordering of quartz. (a) Enthalpy (left axis) and density (right axis) of irradiated $\alpha$-quartz as a function of the deposited energy (DE). (b) Evolution of the structure of $\alpha$-quartz following different amounts of (radiation) energy deposition, namely, $0 \mathrm{eV} /$ atom, $1.8 \mathrm{eV} /$ atom, and $18 \mathrm{eV} /$ atom.

vacancy defects, etc. The defects formed herein include, amongst others, overcoordinated and undercoordinated $\mathrm{Si}$ and $\mathrm{O}$ species, stretched bonds or angles, edge-sharing $\mathrm{Si}$ tetrahedra, mis-sized silicate rings, etc. [25]. As shown in Fig. 1(b), the percolation of such defects results in the complete disordering of the network-note that, in such amorphous networks, the very notion of a traditional crystallographic defect eventually becomes ill-defined. To ensure that the properties of the irradiated quartz samples do not significantly depend on the incident energy per neutron, but rather on the total deposited energy, the present methodology was repeated for varying system sizes and neutron energies. We observe that the obtained results do not exhibit any significant dependencies on either the system size or the incident energy (see Ref. [36]), which suggests a self-similar nature of the damage cascade. It is worth noting that irradiated quartz samples are out of equilibrium and can therefore relax toward more stable configurations upon annealing [41]. However, because of the high viscosity of the system at room temperature, relaxation is kinetically frozen at low temperatures-as observed in the case of silicate glasses [42]. The relaxation of irradiated quartz at different annealing temperatures is presented elsewhere [41].

A visual inspection of the obtained atomic structures reveals that any structural periodicity reminiscent of that of quartz is lost after a deposited energy of around $3 \mathrm{eV} /$ atom. A detailed analysis of such disordering in terms of the short-range and medium-range defects has been presented elsewhere [43]. The disordering is apparent from the examination of the pair distribution functions (PDFs) of quartz, which, with increasing dosage of deposited energy, denote a gradual loss of long-range order [Fig. 2(a)]. This loss of periodicity, in the limit, is in agreement with electron diffraction analysis of irradiated quartz $[8,16]$. Furthermore, as shown in Fig. 2(b), irradiation also results in a decrease of the $\mathrm{Si}-\mathrm{O}-\mathrm{Si}$ bond angle, in agreement with experiments [37]. Altogether, the methodology and the reactive interatomic potential used herein (see Sec. II) offer a realistic description of the influences of irradiation on $\alpha$-quartz and, as such, provide a reliable basis to assess the topography of the enthalpy landscape and compare irradiation-induced disordering to that observed upon thermal vitrification.

We now compare the structure of irradiated quartz, after the saturation of its density and enthalpy, to that of glassy silica. First, we note that, as expected, the density of fully irradiated quartz is fairly similar to that of glassy silica [38], i.e., around $2.2 \mathrm{~g} / \mathrm{cm}^{3}$. Second, we observe that the PDF of irradiated quartz shows the typical features of a glassy structure, that is, well-defined short-range order and poorly defined long-range order. Nevertheless, the PDF of irradiated quartz differs from that of glassy silica: (1) At short distances, the peaks appear broader than in glassy silica, (2) a peak around $2 \AA$ that is absent in glassy silica is observed (N.B.: the existence of such a peak has been suggested experimentally [44]), and (3) at larger distances (>3 $\AA$ ), the peaks are not as well defined as in glassy silica. Points (1) and (2) arise from the existence in the atomic network of undercoordinated or overcoordinated $\mathrm{Si}$ and $\mathrm{O}$ species, which are virtually absent in glassy silica [25,31], while point (3) suggests that the medium-range order-i.e., structural correlations at distances larger than the first coordination shells — of irradiated quartz is less pronounced than that of glassy silica. Similarly, as shown in Fig. 2(b), both the $\mathrm{O}-\mathrm{Si}-\mathrm{O}$ and $\mathrm{Si}-\mathrm{O}-\mathrm{Si}$ bond angle distributions are 

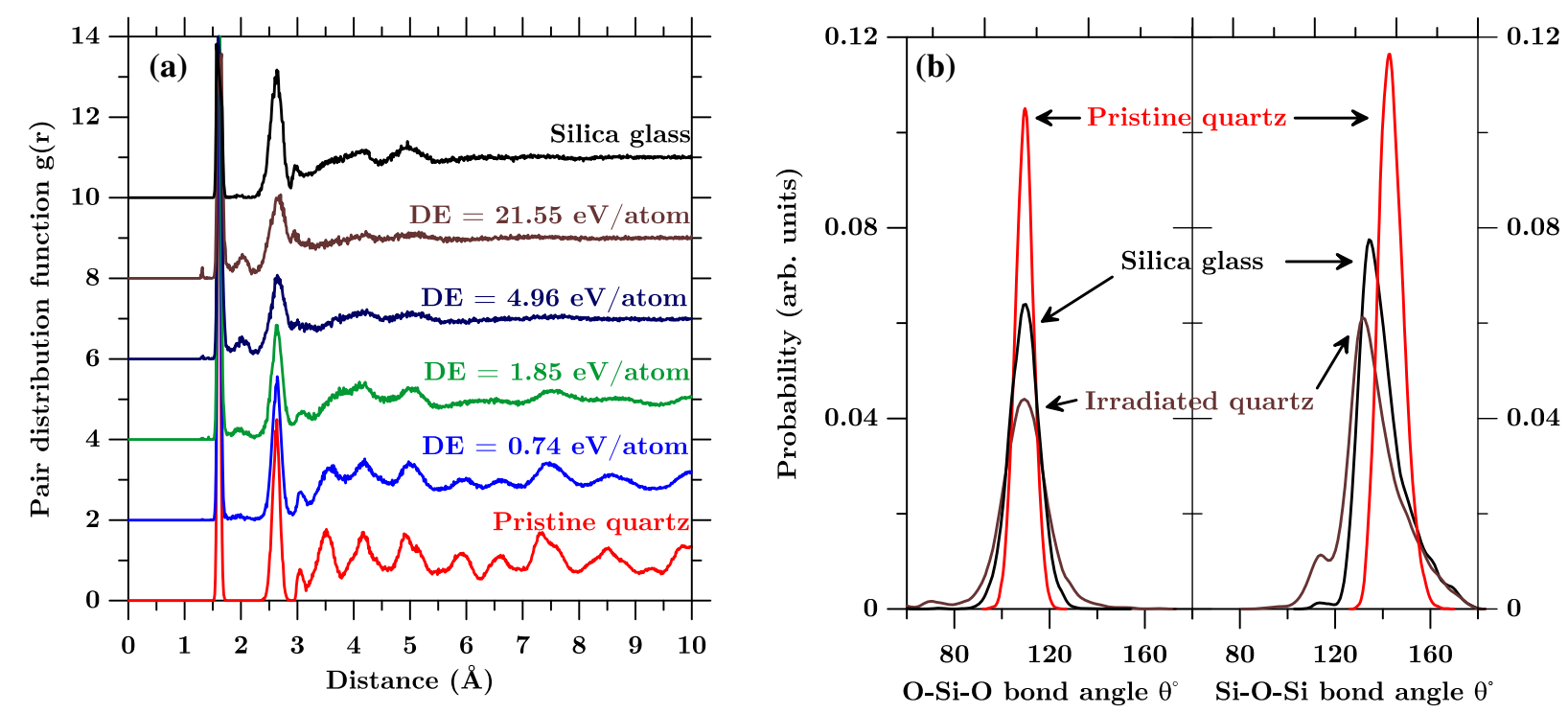

FIG. 2. Comparison between irradiated quartz and glassy silica. (a) Pair distribution functions of pristine $\alpha$-quartz, irradiated quartz with increasing dosages of $\mathrm{DE}$, and glassy silica. (b) Intratetrahedral (O-Si-O) and intertetrahedral (Si-O-Si) bond angle distributions in pristine $\alpha$-quartz, glassy silica, and fully irradiated quartz.

broader in irradiated quartz. Note that a detailed discussion on the origin of these defects and how they differ from the typical structure of glassy silica has been presented elsewhere [45]. Overall, although irradiated quartz features a disordered structure, the short- and medium-range order of irradiated quartz appear to be more disordered than in glassy silica.

Having established that irradiated quartz differs from glassy silica, as obtained by quenching a silica melt with a finite cooling rate, we now investigate to what extent this result depends on the thermal history of the glass. Indeed, as nonequilibrium materials, the properties of glasses at fixed thermodynamic conditions depend on the thermodynamic path followed since their last equilibrium configuration, e.g., their thermal and pressure histories $[10,46]$. Note that, although the short- and medium-range order structure (bond lengths, coordination numbers, PDF, structure factor, etc.) only weakly depends on the thermal history, the thermodynamic properties, such as the density or thermal expansion, are found to be highly sensitive to the thermal path followed upon cooling [37,38]. The probability of each final configuration can be assessed from the knowledge of the enthalpy landscape, that is, the hypersurface of the enthalpy as a function of the volume and atomic positions [41,47]. The enthalpy landscape contains various energy basins, or local minima, that are connected via channels. At high temperatures, in the liquid state, the system can explore several basins and, as such, is ergodic and at equilibrium. However, as the temperature decreases, the probability of transitioning between distinct basins decreases, which ultimately results in a loss of ergodicity. Finally, once in the glassy state, the system is trapped in a local minimum of the enthalpy landscape. Mauro et al. showed that only a small fraction of the enthalpy landscape basins are accessible to glasses, regardless of their thermal history-e.g., their cooling rate or annealing duration [48]. The configurations associated with these accessible basins are called "allowable glasses." In contrast, all the other disordered configurations, which are not accessible via traditional thermal pathways, are referred to as "forbidden glasses" [48]. Accessing these forbidden configurations requires the application of an external stimulus, such as high pressure [49] or ion-exchange treatment [42,50]. From a practical standpoint, these systems exhibit properties that are notably different from those of their "allowable" counterparts (e.g., density, hardness, and heat capacity [49]). In the following, we investigate if irradiated quartz, before or after defect saturation, lies in forbidden or allowable regions of the enthalpy landscape.

Figure 3(a) shows the ground-state enthalpy $H_{0}$ (see Sec. II) of $\alpha$-quartz and glassy silica as a function of temperature. As expected, we observe that, in the case of the crystal, $H_{0}$ remains fairly constant at low temperatures, until the melting temperature $T_{m}$ is reached. At $T_{m}$, a firstorder transition is observed, which manifests as a discontinuity of the enthalpy and density. Similarly, $H_{0}$ also remains fairly constant at low temperatures in glassy silica. In agreement with the original postulate of Zachariasen [51], the enthalpy of glassy silica has the same order of magnitude as that of its crystalline counterpart, being only slightly higher because of the absence of long-range order. However, as expected, no clear phase transition is observed in glassy silica. Rather, in the vicinity of the glass transition temperature $T_{g}$, $H_{0}$ continuously increases and gradually reaches the same slope as that observed for liquid silica, as melted from the crystal. In this regime $\left(T_{g} \approx 2500 \mathrm{~K}<T<T_{m} \approx 4000 \mathrm{~K}\right)$, 

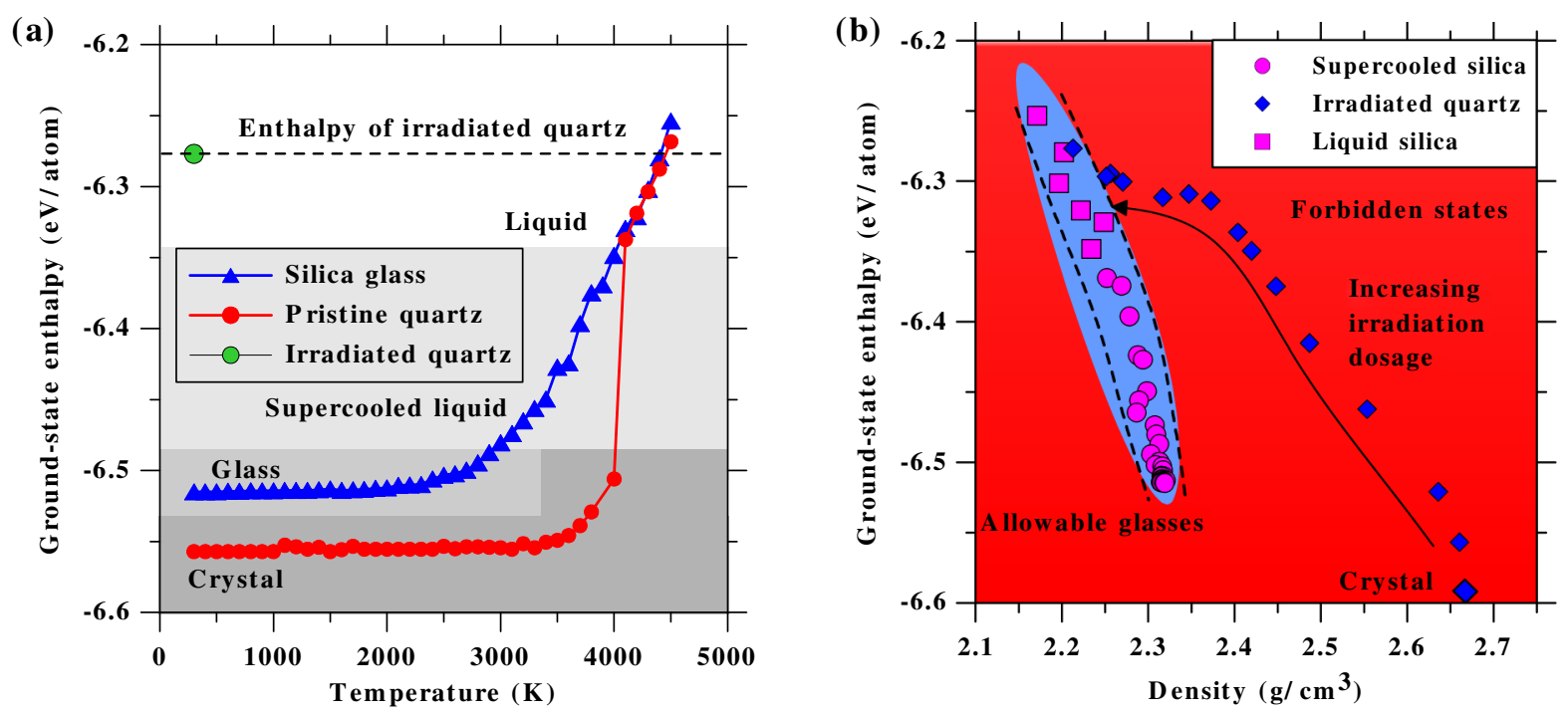

(c)
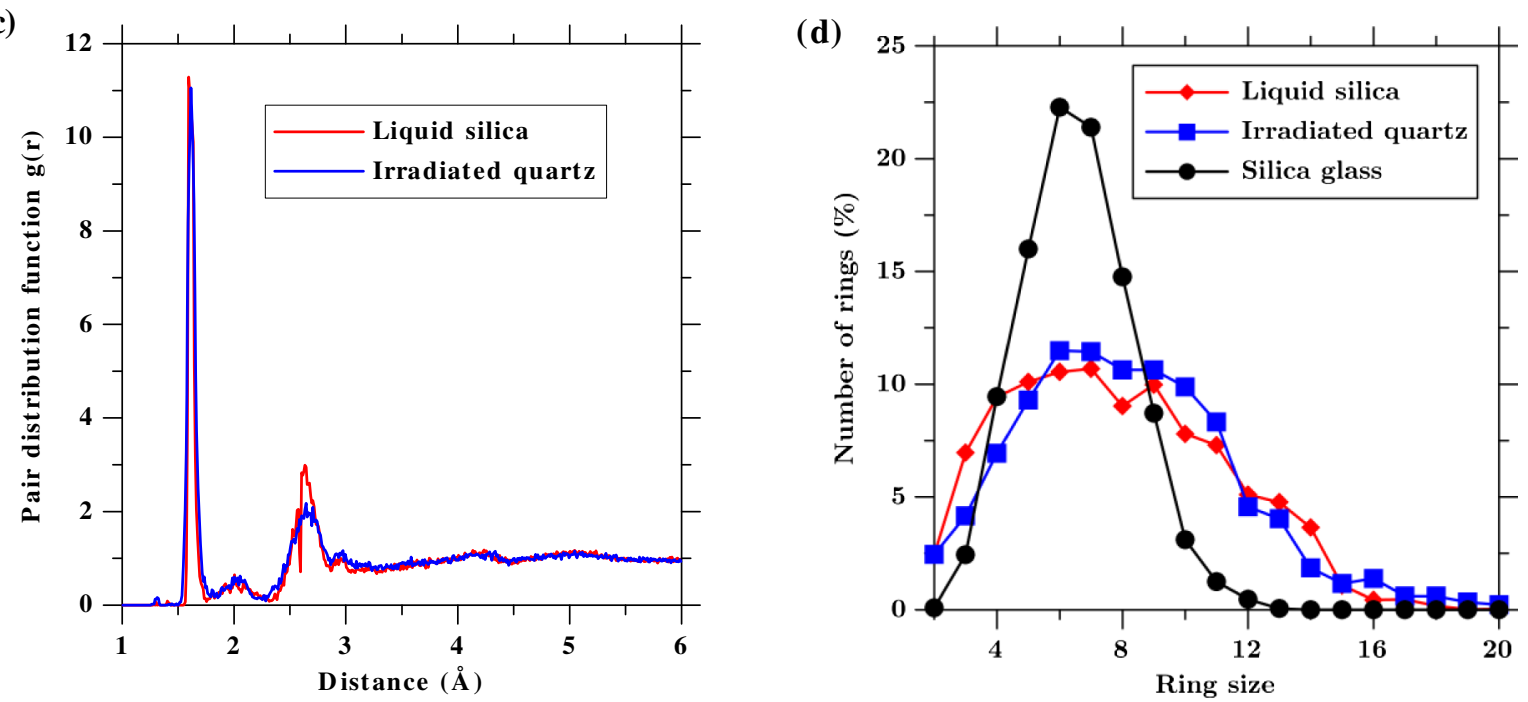

FIG. 3. Comparison of the effects of irradiation and temperature. (a) Ground-state enthalpy of pristine $\alpha$-quartz and glassy silica as a function of temperature, compared with that of fully irradiated quartz at $300 \mathrm{~K}$. The colored areas indicate the extent of the crystalline, glassy, supercooled liquid, and liquid states. (b) Positions of partially irradiated quartz samples in the ground-state enthalpy-density space, for increasing deposited energies. These data are compared to the ground-state enthalpy or density of $\alpha$-quartz and liquid or supercooled silica at different temperatures. The light-blue and red background colors indicate the extent of the regions of allowable glasses and forbidden states, respectively. (c) Pair distribution function, $g(r)$, of fully irradiated quartz, compared to that of a silica liquid hyperquenched from $4400 \mathrm{~K}$. (d) Ring-size distribution of fully irradiated quartz, compared to that of a silica liquid hyperquenched from $4400 \mathrm{~K}$ and glassy silica. (For reference, the ground-state enthalpy of quartz with respect to the deposited energy is presented in Fig. S1 of Ref. [36].)

silica reaches a supercooled liquid state, which is ergodic and at metastable equilibrium.

As shown in Fig. 3(a), the $H_{0}$ of fully irradiated quartz is significantly higher than that of $\alpha$-quartz and glassy silica at room temperature, as it is indeed more disordered than glassy silica. We note that the $H_{0}$ of fully irradiated quartz corresponds to that of a silica melt at around $4400 \mathrm{~K}$. This suggests that irradiated quartz is equivalent to a "frozen liquid" with a "fictive temperature" $T_{f}$ of $4400 \mathrm{~K}$. Note that such concepts of fictive temperature are typically used to describe the relaxation state of a glass; namely, a glass with a given $T_{f}$ shows the same structure as an equilibrium supercooled liquid at this temperature [10]. Namely, glass formed with lower cooling rates can relax to lower energy states and, as such, tend to feature lower values of $T_{f}$. In the following, we investigate whether the irradiation-induced disordering of quartz is equivalent to glasses featuring increasing $T_{f}$.

To answer this question, we track the states of irradiated and vitrified $\mathrm{SiO}_{2}$ in the enthalpy-density $\left(H_{0}-\rho\right)$ space. 
Although we note that mere knowledge of $H_{0}$ and $\rho$ is not sufficient to fully characterize out-of-equilibrium materials, this choice is motivated by the fact that short-range interactions constitute the main contribution to $H_{0}$, so it offers a good metric to characterize the short-range order of the system. Conversely, $\rho$ is a more complex property that depends on fine structural arrangements, including longerrange features such as dihedral angle, ring-size distribution, etc. As such, density can be used as a metric to discriminate systems featuring a similar short-range order but different medium- and long-range orders [46].

Figure 3(b) shows the states of liquid and supercooled liquid silica with varying $T_{f}$ in the enthalpy-density space. (For reference, the ground-state enthalpy of quartz with respect to the deposited energy is presented in Fig. S1 in Ref. [36]). We observe that these configurations form a line in the $H_{0}-\rho$ space; that is, $H_{0}$ decreases linearly with $\rho$. As such, this line-and its extrapolation to lower and higher temperatures - indicates the extent of the allowable domain, that is, the set of the atomic configurations of allowable glasses with varying $T_{f}$, which can be formed by simply cooling a melt. The two theoretical limits of this domain correspond to the following: (1) at high temperatures-a hyperquenched liquid, i.e., a $\mathrm{SiO}_{2}$ melt that is instantly quenched to zero temperature, and (2) at low temperatures-an "ideal glass" [12], i.e., a supercooled liquid that reaches its theoretical Kauzmann temperature [52].

We now assess the configurational states explored by irradiated quartz upon different dosages of irradiation. As shown in Fig. 3(b), irradiation induces an increase of $H_{0}$ and a decrease of $\rho$. We observe that, as irradiation-induced defects start to accumulate, the thermodynamic states achieved by irradiated quartz lie outside of the region of allowable glasses. This shows that, prior to defect saturation, irradiated quartz explores forbidden states of the enthalpy landscape that cannot be accessed by cooling a melt. More specifically, for a given $\rho$, irradiated quartz shows a higher $H_{0}$ than supercooled liquid silica, in agreement with the fact that irradiated quartz contains more short-range order defects than glassy silica. It should be noted that before the saturation of defects, the system does not exhibit a two-phase behavior-that is, partly crystalline and partly disordered-and becomes fully disordered well before it reaches an allowable glass (see Ref. [36]). Altogether, this demonstrates that irradiationinduced disordering fundamentally differs from that observed upon vitrification and cannot be understood as simply an increase of fictive temperature.

Interestingly, we observe that, eventually, irradiationinduced damage saturates when the irradiated system reaches the state of an allowable silica liquid experiencing a temperature of $4400 \mathrm{~K}$ in the $H_{0}-\rho$ space [see Fig. 3(b)]. To ascertain whether fully irradiated quartz indeed presents a structure similar to that of a frozen liquid, the PDF of fully irradiated quartz is compared to that of a silica liquid at $4400 \mathrm{~K}$. To enable a meaningful comparison, the liquid is instantaneously quenched to zero temperature (i.e., hyperquenched) before the PDF is calculated. As shown in Fig. 3(c), we observe a good agreement between the PDFs of fully irradiated quartz and liquid silica. In particular, we note that the first two peaks show a similar position and shape, which shows that the defected short-range order of fully irradiated quartz is similar to that of the silica liquid. We also observe that the peaks at larger distance show good agreement, which suggests that fully irradiated quartz and liquid silica share similar medium- and long-range order, or absence thereof. The medium- and long-range order are more accurately captured by the ring-size distribution (RSD) within the silicate network (where rings are defined as the shortest closed paths made of $\mathrm{Si}-\mathrm{O}$ bonds within the atomic network, see Sec. II), which is shown in Fig. 3(d) for fully irradiated quartz, liquid silica, and glassy silica. We observe that glassy silica exhibits a relatively sharp RSD centered around an average size of 6.5, in agreement with experiment [53]. In contrast, the RSDs of irradiated quartz and liquid silica significantly differ from that of glassy silica as they show broader distributions, centered around larger average rings (around 8). In turn, the RSDs of irradiated quartz and liquid silica share a large degree of similarity, which further demonstrates the striking structural concordance between irradiated quartz and liquid silica. Altogether, this shows that fully irradiated quartz is effectively a frozen allowable liquid, that is, a disordered system that is obtained by instantaneously hyperquenching a melt. It is worth noting that, from the viewpoint of topological constraint theory, irradiation results in the breakage of the weak $\mathrm{Si}-\mathrm{O}-\mathrm{Si}$ bond-bending constraints, which are also thermally broken in silicate liquids [54]. This further supports the equivalence between irradiated quartz and a hyperquenched liquid.

\section{DISCUSSION}

We now discuss the origin of the saturation of damage as irradiated quartz achieves the structure of an allowable frozen liquid. Such behavior can be understood by considering how irradiation affects the configurational position of the system in the enthalpy landscape of $\mathrm{SiO}_{2}$, as depicted in Fig. 4(a). The energy basin corresponding to the crystalline state is typically very narrow [12]. The system can exit this energy basin if the deposited radiation energy reaches a threshold value $(28.9 \mathrm{eV}$ for oxygen and $70.5 \mathrm{eV}$ for silicon in $\alpha$-quartz [25]) permitting one to overcome the surrounding energy barriers, thereby allowing the system to reach another basin corresponding to a defective structure. At this stage, because of its vicinity to the initial crystalline configuration, the local enthalpy landscape remains rough and features high-energy barriers. Additional deposited energy allows the system to reach higher energy basins associated with other forbidden configurations. Finally, as 

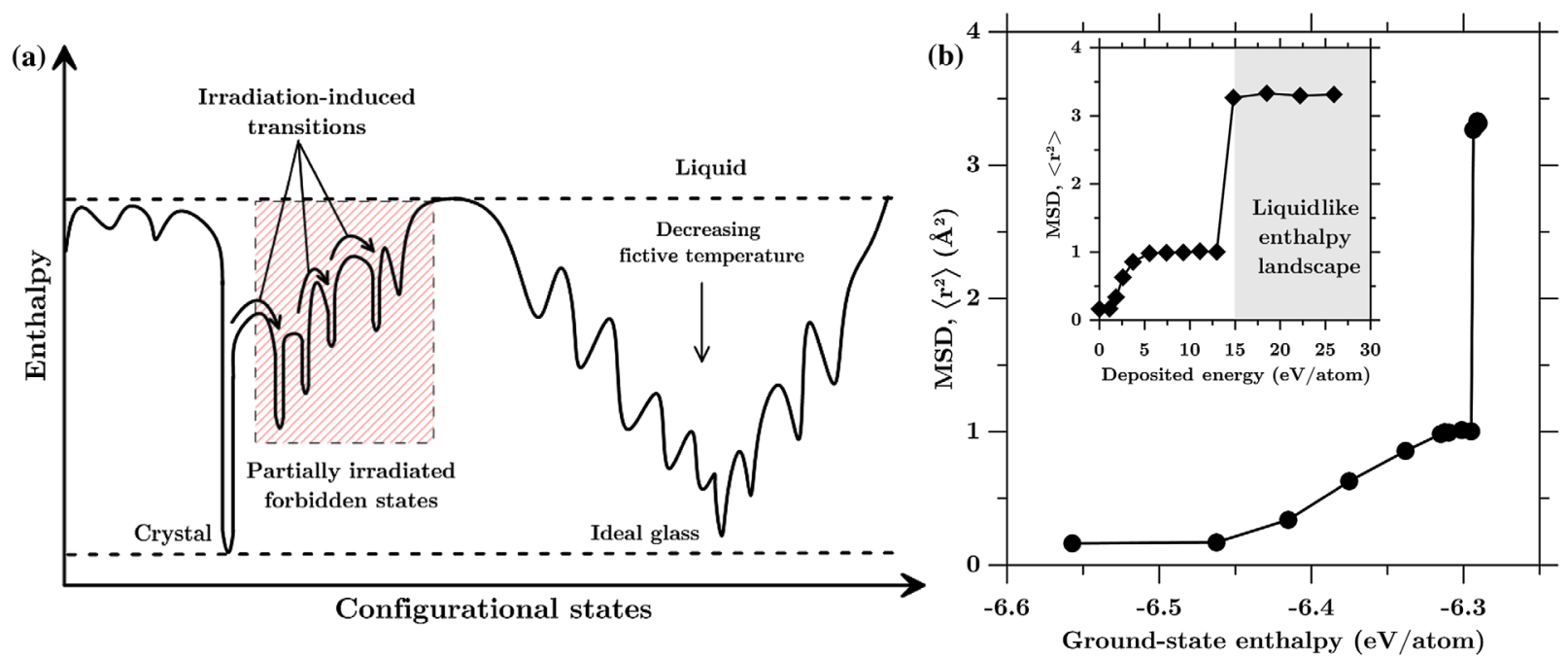

FIG. 4. Topography of the enthalpy landscape. (a) Schematic of the enthalpy landscape explored upon irradiation. The $x$ axis represents all the configurational states. The red box indicates the extent of the forbidden region that is not accessible by conventional thermal pathways. (b) Atomic MSD explored by the atoms during 100 ps of dynamics following a similar energy bump in pristine, partially irradiated, and fully irradiated quartz samples with respect to the ground-state enthalpy. The inset shows the same values of MSD with respect to the deposited energy. The shaded region indicates the extent of the liquidlike region of the enthalpy landscape, for which irradiation-induced damage saturates.

the system experiences enough deposited energy to reach an energy state comparable to that of a liquid, it eventually encounters lower-energy barriers [55].

To establish this mechanism, we estimate the height of the energy barriers by computing the atomic mean-square distance (MSD) between irradiated quartz configurations at $1500 \mathrm{~K}$ and the corresponding inherent structures, i.e., after enthalpy minimization (see Sec. II). By capturing the extent of the thermally induced atomic motion, such a metric has been shown to accurately describe the height of the energy barriers locally sampled in the enthalpy landscape [55]. Namely, lower-energy barriers result in higher atomic motion and, as such, higher values of MSDs. As shown in Fig. 4(b), we observe a sharp increase of the MSD as the system reaches a frozen-liquid configuration. This denotes a sudden change of the topography of the enthalpy landscape, that is, a decrease in the height of the energy barriers. In turn, the roughness of the enthalpy landscape controls the extent of possible reorganizations for the network. At low deposited energy, high-energy barriers prevent any transition to other energy basins and, thereby, significantly limit the extent of possible structural relaxation. As such, irradiation-induced defects accumulate. In contrast, once the system reaches a frozen-liquid state, lower-energy barriers facilitate transitions between basins so that any further deposited energy will significantly move the system away from its original energy basin. This ability to freely sample many energy basins allows the system to relax, that is, to feature local structural reorganizations. Therefore, the system is able to relax any additional unfavorable structural defects that would otherwise form because of further irradiation. Consequently, this liquidlike configuration constitutes the upper limit of irradiation-induced damage. From a practical standpoint, the equivalence between irradiated materials and hyperquenched liquids could be used as a basis to predict the swelling of mineral when subjected to irradiation, that is, by comparing the density of the pristine crystal and of its isochemical liquid. Hence, this approach can provide a means to identify radiation-resistant materials.

\section{OUTLOOK}

Overall, our analysis highlights the fundamental importance of the underlying enthalpy, which provides a consistent explanation for the accumulation and saturation of structural defects in quartz. In a similar fashion as the roughness of the enthalpy landscape explored in the supercooled liquid state controls the fragility of glasses (i.e., the extent of deviation from an ideal Arrhenius behavior for the viscosity at the vicinity of $T_{g}[12]$ ), it is expected that the roughness of the enthalpy landscape around the crystalline state will control the value of the minimum threshold energy that can induce some permanent damage, as well as the ability for the system to recrystallize or not upon thermal annealing. As such, elucidating the topography of the enthalpy landscape, i.e., the positions of basin minima and the height of saddle points, appears to be key to understanding and predicting a mineral's resistance to irradiation.

\section{ACKNOWLEDGMENTS}

This research was performed using funding received from the Department of Energy Office of Nuclear Energy's 
Nuclear Energy University Programs. The authors also acknowledge financial support for this research provided by the Oak Ridge National Laboratory operated for the U.S. Department of Energy by UT-Battelle (LDRD Grants No. 4000132990 and No. 4000143356), the National Science Foundation (CMMI: 1235269, Grant No. 1253269), Federal Highway Administration (DTFH61-13-H-00011), and the University of California, Los Angeles (UCLA). This manuscript has been coauthored by UT-Battelle, LLC under Contract No. DEAC05-00OR22725 with the U.S. Department of Energy.

[1] K. Trachenko, Understanding Resistance to Amorphization by Radiation Damage, J. Phys. Condens. Matter 16, R1491 (2004).

[2] K. E. Sickafus, R. W. Grimes, J. A. Valdez, A. Cleave, M. Tang, M. Ishimaru, S. M. Corish, C. R. Stanek, and B. P. Uberuaga Radiation-Induced Amorphization Resistance and Radiation Tolerance in Structurally Related Oxides, Nat. Mater. 6, 217 (2007).

[3] K. E. Sickafus et al. Radiation Tolerance of Complex Oxides, Science 289, 748 (2000).

[4] M. Schulz, Ion Implantation: A Useful Tool for Semiconductor Research, Appl. Phys. 4, 91 (1974).

[5] A. M. Stoneham, J. R. Matthews, and I. J. Ford, Innovative Materials for Fusion Power Plant Structures: Separating Functions, J. Phys. Condens. Matter 16, S2597 (2004).

[6] R. Devanathan, Ph.D. thesis, Northwest University, 1993.

[7] M. R. Pascucci, J. L. Hutchison, and L. W. Hobbs, The Metamict Transformation in Alpha-Quartz, Radiat. Eff. 74, 219 (1983).

[8] S. Weissmann and K. Nakajima, Defect Structure and Density Decrease in Neutron-Irradiated Quartz, J. Appl. Phys. 34, 611 (1963).

[9] J. M. Delaye and D. Ghaleb, Molecular Dynamics Simulation of Low-Energy Atomic Displacement Cascades in a Simplified Nuclear Glass, J. Nucl. Mater. 244, 22 (1997).

[10] A. K. Varshneya, Fundamentals of Inorganic Glasses (Elsevier, New York, 2013).

[11] J. F. Denatale and D. G. Howitt, A Mechanism for Radiation Damage in Silicate Glasses, Nucl. Instrum. Methods Phys. Res., Sect. B 1, 489 (1984).

[12] P. G. Debenedetti and F. H. Stillinger, Supercooled Liquids and the Glass Transition, Nature (London) 410, 259 (2001).

[13] W. J. Weber, Models and Mechanisms of IrradiationInduced Amorphization in Ceramics, Nucl. Instrum. Methods Phys. Res., Sect. B 166-167, 98 (2000).

[14] K. E. Sickafus, J. A. Valdez, J. R. Williams, R. W. Grimes, and H. T. Hawkins, Radiation Induced Amorphization Resistance in $\mathrm{A}_{2} \mathrm{O}_{3}-\mathrm{BO}_{2}$ oxides, Nucl. Instrum. Methods Phys. Res., Sect. B 191, 549 (2002).

[15] L. W. Hobbs, The Role of Topology and Geometry in the Irradiation-Induced Amorphization of Network Structures, J. Non-Cryst. Solids 182, 27 (1995).

[16] I. Pignatelli et al. Direct Experimental Evidence for Differing Reactivity Alterations of Minerals Following
Irradiation: The Case of Calcite and Quartz, Sci. Rep. 6, 20155 (2016).

[17] L. W. Hobbs, Topology and Geometry in the IrradiationInduced Amorphization of Insulators, Nucl. Instrum. Methods Phys. Res., Sect. B 91, 30 (1994).

[18] M. R. Levy, R. W. Grimes, and K. E. Sickafus, Disorder Processes in $\mathrm{A}^{3+} \mathrm{B}^{3+} \mathrm{O}_{3}$ Compounds: Implications for Radiation Tolerance, Philos. Mag. 84, 533 (2004).

[19] S. O. Kucheyev, J. S. Williams, J. Zou, and C. Jagadish, Dynamic Annealing in III-Nitrides under Ion Bombardment, J. Appl. Phys. 95, 3048 (2004).

[20] S. X. Wang, G. R. Lumpkin, L. M. Wang, and R. C. Ewing, Ion Irradiation-Induced Amorphization of Six Zirconolite Compositions, Nucl. Instrum. Methods Phys. Res., Sect. B 166, 293 (2000).

[21] R. Devanathan, J. N. Mitchell, K. E. Sickafus, W. J. Weber, and M. Nastasi, Radiation Response of $\mathrm{FeTiO}_{3}, \mathrm{MgTiO}_{3}$, and $\alpha-\mathrm{Al}_{2} \mathrm{O}_{3}$, Mater. Sci. Eng. A 253, 131 (1998).

[22] R. C. Ewing, L. M. Wang, and W. J. Weber, Amorphization of Complex Ceramics by Heavy-Particle Irradiations, in MRS Proceedings (Cambridge University Press, Cambridge, England, 1994), Vol. 373, p. 347.

[23] R. Devanathan, L. R. Corrales, W. J. Weber, A. Chartier, and C. Meis, Molecular Dynamics Simulation of Disordered Zircon, Phys. Rev. B 69, 064115 (2004).

[24] S. Plimpton, Fast Parallel Algorithms for Short-Range Molecular Dynamics, J. Comput. Phys. 117, 1 (1995).

[25] B. Wang, Y. Yu, I. Pignatelli, G. Sant, and M. Bauchy, Nature of Radiation-Induced Defects in Quartz, J. Chem. Phys. 143, 024505 (2015).

[26] W. J. Weber, Radiation-Induced Defects, and Amorphization in Zircon, J. Mater. Res. 5, 2687 (1990).

[27] W. G. Hoover, Canonical Dynamics: Equilibrium PhaseSpace Distributions, Phys. Rev. A 31, 1695 (1985).

[28] G. Roma, Y. Limoge, and S. Baroni, Oxygen Self-Diffusion in $\alpha$-Quartz., Phys. Rev. Lett. 86, 4564 (2001).

[29] A. C. Van Duin, S. Dasgupta, F. Lorant, and W. A. Goddard, ReaxFF: A Reactive Force Field for Hydrocarbons, J. Phys. Chem. A 105, 9396 (2001).

[30] H. Manzano, S. Moeini, F. Marinelli, A. C. T. van Duin, F.-J. Ulm, and R. J.-M. Pellenq Confined Water Dissociation in Microporous Defective Silicates: Mechanism, Dipole Distribution, and Impact on Substrate Properties, J. Am. Chem. Soc. 134, 2208 (2012).

[31] Y. Yu, B. Wang, M. Wang, G. Sant, and M. Bauchy, Revisiting Silica with ReaxFF: Towards Improved Predictions of Glass Structure and Properties via Reactive Molecular Dynamics, J. Non-Cryst. Solids 443, 148 (2016).

[32] S. Goverapet Srinivasan, and A. C. T. van Duin, MolecularDynamics-Based Study of the Collisions of Hyperthermal Atomic Oxygen with Graphene Using the ReaxFF Reactive Force Field, J. Phys. Chem. A 115, 13269 (2011).

[33] T.P. Senftle et al. The ReaxFF Reactive Force-Field: Development, Applications and Future Directions, npj Comput. Mater. 2, 15011 (2016).

[34] M. Parrinello and A. Rahman, Polymorphic Transitions in Single Crystals: A New Molecular Dynamics Method, J. Appl. Phys. 52, 7182 (1981).

[35] S. Le Roux and P. Jund, Ring Statistics Analysis of Topological Networks: New Approach and Application to 
Amorphous $\mathrm{GeS}_{2}$ and $\mathrm{SiO}_{2}$ Systems, Comput. Mater. Sci. 49, 70 (2010).

[36] See Supplemental Material at http://link.aps.org/ supplemental/10.1103/PhysRevX.7.031019 for more details about the simulation methodology.

[37] I. Simon, Structure of Neutron-Irradiated Quartz and Vitreous Silica, J. Am. Ceram. Soc. 40, 150 (1957).

[38] N.P. Bansal and R. H. Doremus, Handbook of Glass Properties. (Elsevier, New York, 2013).

[39] W. Primak, Fast-Neutron-Induced Changes in Quartz and Vitreous Silica, Phys. Rev. 110, 1240 (1958).

[40] V. N. Bykov, A. V. Denisov, V. B. Dubrovskii, V. V. Korenevskii, G. K. Krivokoneva, and L. P. Muzalevskii Effect of Irradiation Temperature on the Radiation Expansion of Quartz, Sov. At. Energy 51, 593 (1981).

[41] J. C. Mauro, R. J. Loucks, and J. Balakrishnan, Split-Step Eigenvector-Following Technique for Exploring Enthalpy Landscapes at Absolute Zero, J. Phys. Chem. B 110, 5005 (2006).

[42] M. Wang and M. Bauchy, Ion-Exchange Strengthening of Glasses: Atomic Topology Matters, arXiv:1505.07880.

[43] N. M. A. Krishnan, B. Wang, Y. Le Pape, G. Sant, and M. Bauchy, Irradiation-Driven Amorphous-to-Glassy Transition in Quartz: The Crucial Role of the Medium-Range Order in Crystallization, (unpublished).

[44] E. Lorch, Neutron Diffraction by Germania, Silica and Radiation-Damaged Silica Glasses, J. Phys. C 2, 229 (1969).

[45] N. A. Krishnan, B. Wang, Y. Le Pape, G. Sant, and M. Bauchy, Irradiation- vs. Vitrification-Induced Disordering: The Case of $\alpha$-Quartz and Glassy Silica, J. Chem. Phys. 146, 204502 (2017).
[46] M. M. Smedskjaer, M. Bauchy, J. C. Mauro, S. J. Rzoska, and M. Bockowski, Unique Effects of Thermal and Pressure Histories on Glass Hardness: Structural and Topological Origin, J. Chem. Phys. 143, 164505 (2015).

[47] J. C. Mauro, R. J. Loucks, A. K. Varshneya, and P. K. Gupta, Enthalpy Landscapes and the Glass Transition, in, Scientific Modeling and Simulations, edited by S. Yip and T. D. Rubia (Springer Netherlands, 2008), pp. 241-281.

[48] J. C. Mauro and R. J. Loucks, Forbidden Glasses and the Failure of Fictive Temperature, J. Non-Cryst. Solids 355, 676 (2009).

[49] M. N. Svenson, J. C. Mauro, S. J. Rzoska, M. Bockowski, and M. M. Smedskjaer, Accessing Forbidden Glass Regimes through High-Pressure Sub-Tg Annealing, Sci. Rep. 7, 46631 (2017).

[50] M. Bauchy, Structural, Vibrational, and Thermal Properties of Densified Silicates: Insights from Molecular Dynamics, J. Chem. Phys. 137, 044510 (2012).

[51] W. H. Zachariasen, The Atomic Arrangement in Glass, J. Am. Chem. Soc. 54, 3841 (1932).

[52] J. C. Mauro, Through a Glass, Darkly: Dispelling Three Common Misconceptions in Glass Science, Int. J. Appl. Glass Sci. 2, 245 (2011).

[53] P. Y. Huang et al. Direct Imaging of a Two-Dimensional Silica Glass on Graphene, Nano Lett. 12, 1081 (2012).

[54] B. Wang, N. M. A. Krishnan, Y. Yu, M. Wang, Y. Le Pape, G. Sant, and M. Bauchy Irradiation-Induced Topological Transition in SiO 2: Structural Signature of Networks' Rigidity, J. Non-Cryst. Solids 463, 25 (2017).

[55] S. Sastry, P. G. Debenedetti, and F. H. Stillinger, Signatures of Distinct Dynamical Regimes in the Energy Landscape of a Glass-Forming Liquid, Nature (London) 393, 554 (1998). 